\title{
El programa nacional para el diagnóstico y tratamiento de depresión en atención primaria: una evaluación necesaria
}

\author{
RUBÉN ALVARADO ${ }^{1}$, GRACIELA ROJAS ${ }^{2}$
}

'Escuela de Salud Pública, Facultad de Medicina, Universidad de Chile. ${ }^{2}$ Departamento de

Psiquiatría y Salud Mental, Hospital Clínico, Universidad de Chile.

Proyecto FONDECYT 1100205

Recibido el 6 de octubre de 2010, aceptado el 23 de marzo de 2011

Correspondencia a: Rubén Alvarado

Escuela de Salud Pública, Facultad de Medicina, Universidad de Chile. Independencia 938 . Santiago. Chile

E-mail: ralvarado@med. uchile.cl

\section{Evaluation of the program for detection and treatment of depression in Chilean primary health care centers}

Background: Depression is highly prevalent among Chilean adults, therefore a primary care treatment program was devised. Aim: To evaluate the Program for detection, diagnosis and integral treatment of depression, implemented in Chilean Primary Health Care Centers. Material and Methods: A three and six months followup study was conducted among women admitted to the program at seven primary health care centers in two regions of Chile. Socio-demographic data, treatments provided and compliance with the program were assessed. A clinical standardized interview (with ICD-10) was carried out and the intensity of depressive symptoms was evaluated using Beck Depression Inventory (BDI). Results: The initial sample was composed by 201 women aged 25 to 65 years. At 6 months, 35\% were lost from follow up. Depression was mild, moderate or severe in 12, 63 and $25 \%$ of women, respectively. The severity of depression was underestimated by primary care teams. Among women with regular and irregular attendance to medical appointments and those that abandoned treatment, BDI score decreased by 35, 37 and 13\%, respectively. Conclusions: This primary care program for depression was effective to reduce BDI scores. Diagnostic precision of primary care teams must be improved.

(Rev Med Chile 2011; 139: 592-599).

Key words: Depression; Primary health care; Questionnaires.
L os trastornos depresivos son uno de los principales problemas de salud de nuestra población (con una prevalencia cercana al $10 \%$ en la población mayor de 15 años $)^{1,2}$. Estos cuadros han sido reconocidos como una de las causas más importantes de pérdida de años de vida saludable, que en el caso de las mujeres llega a ocupar el segundo lugar en nuestro país ${ }^{3}$.

Las investigaciones en población consultante de centros de atención primaria han revelado que una proporción alta, entre $30 \%$ y $50 \%$, presenta un trastorno emocional, ya sea como motivo de consulta o en forma "encubierta" a través de síntomas de carácter somático ${ }^{4}$.
Existe evidencia que demuestra que los trastornos depresivos tienen una buena respuesta a los tratamientos convencionales con fármacos antidepresivos, psicoterapia o ambos ${ }^{5,6}$. Esta eficacia es aun mayor cuando las atenciones se organizan en torno a programas, con equipos multiprofesionales y acciones coordinadas, que incluyen educación del paciente y su familia, así como el control y seguimiento de las indicaciones ${ }^{7,8}$, tal como se demuestra en recientes revisiones sistemáticas ${ }^{9,10}$.

En nuestro país, entre los años 1999 y 2001 se realizó un ensayo clínico controlado en centros de atención primaria que permitió evaluar la efectividad de un programa de atención para la 
depresión, que incluía la participación en un grupo estructurado de psicoeducación, el monitoreo sistemático de la evolución clínica y el tratamiento farmacológico para los casos más severos. Las personas que recibieron este programa de atención obtuvieron una tasa de recuperación de $70 \%$, en contraste con $30 \%$ observado en el grupo con tratamiento habitual ${ }^{11}$.

Producto de todos estos antecedentes, el Ministerio de Salud implementó el año 2001 el "Programa para la Detección, Diagnóstico y Tratamiento Integral de la Depresión en Atención Primaria", que consiste en un sistema de atención que combina intervenciones médicas y psicosociales, con actividades que incluyen la detección, el diagnóstico, el registro, el tratamiento y el seguimiento de cada caso. Se establece una guía clínica que ordena a través de un algoritmo las diferentes decisiones que los profesionales deberían tomar respecto de los pacientes ${ }^{12}$; y desde el año 2003 este programa se ha extendido a todo el país.

El objetivo de la presente comunicación es informar los resultados de una evaluación de este programa, respecto de la evolución clínica de un grupo de mujeres que ingresaban a tratamiento y de su grado de adherencia a las diferentes intervenciones que en él se realizan.

\section{Material y Método}

Se trata de un estudio prospectivo, basado en una cohorte de una muestra accidental de pacientes que ingresaron al programa entre agosto de 2003 y agosto de 2004, en 7 centros urbanos de atención primaria de las Regiones V y Metropolitana.

Se seleccionaron centros de atención primaria que tuvieran equipos consolidados para la atención de estos pacientes (que llevaran más de un año funcionando, a mediados del año 2003) y que estuviesen dispuestos a colaborar en esta investigación. Previo al ingreso, las mujeres recibieron información sobre el estudio y se solicitó un consentimiento informado. No se registraron rechazos en esta etapa del estudio.

La muestra incluyó a mujeres entre 25 y 65 años de edad ingresadas al Programa en los centros participantes. Sólo se incluyeron mujeres porque estas corresponden a más de $90 \%$ de los casos que ingresan a tratamiento y el haber incorporado ambos sexos hubiese obligado a un análisis de género ${ }^{13}$, reduciendo la factibilidad del estudio. Los criterios de exclusión fueron: estar fuera del rango etario establecido, estar embarazada o haber tenido su parto en los dos meses previos al ingreso a tratamiento, presentar alguna condición que limite la entrevista (retardo mental, deterioro cognitivo, hipoacusia, ceguera) y no tener acceso a teléfono.

La estimación del tamaño muestral se basó en los datos de eficacia del ensayo clínico controlado realizado previamente en Chile $(70 \% \text { vs } 30 \%)^{11}$. Con estos datos, se requerirían 28 casos en cada grupo, para obtener $95 \%$ de confianza y $80 \%$ de poder. Considerando una tasa de abandono del $20 \%$ a los 3 meses (basado en información de un estudio previo de efectividad de este programa, realizado dos años antes durante su fase de puesta en marcha ${ }^{14}$ ), y una tasa de pérdidas de $15 \%$ durante el seguimiento, se requieren 165 casos en total. Finalmente, 201 mujeres ingresaron a la cohorte, 173 accedieron a ser entrevistadas a los 3 meses $(86,1 \%)$ y 166 a los 6 meses $(82,6 \%)$. Es decir, hubo una pérdida de casos de 17,4\% durante el seguimiento.

Se realizó una entrevista estructurada al ingreso, a los 3 y a los 6 meses. Esta información se complementó con los registros del programa, disponibles en cada consultorio. Para mejorar la tasa de seguimiento y la calidad de los datos las mujeres fueron contactadas telefónicamente al mes y a los 2 meses. Todas las entrevistas fueron realizadas por dos profesionales mujeres, especialmente entrenadas para tal efecto.

Se consignaron datos socio-demográficos, se valoró el cumplimiento de los criterios diagnósticos para episodio depresivo según CIE-10 (diferenciando depresión leve, moderada y severa), se midió la intensidad de la sintomatología depresiva, los tratamientos indicados y su cumplimiento, así como la adherencia al programa de tratamiento. La intensidad de los síntomas depresivos se midió con el Inventario de Depresión de Beck (IDB), que es un instrumento auto-aplicado con 21 ítems, ampliamente utilizado en todo el mundo para esta finalidad y cuyas propiedades psicométricas son bien conocidas ${ }^{15-17}$. En un estudio previo con pacientes de este programa, ya lo habíamos utilizado, mostrando buena capacidad para detectar cambios a lo largo del tiempo ${ }^{14}$.

Los datos fueron analizados usando STATA 10.0. El análisis descriptivo se realizó a través de distribución de frecuencias, estimaciones de 
promedios y desviaciones estándar, o medianas, dependiendo del tipo de variables. Para el estudio de la posible asociación entre variables categoriales se usó la prueba de Chi Cuadrado. Para la comparación del puntaje en el IDB se utilizó la prueba de t-Student cuando se trataba de 2 categorías y análisis de varianza cuando eran 3 categorías, en cuyo caso se agregó una prueba de comparaciones múltiples (técnica de Bonferroni).

\section{Resultados}

\section{Características de la muestra}

El grupo tuvo un promedio de edad de 44 años ( de $=11$ años $).$ La mayoría de las mujeres tenía una pareja estable $(47,8 \%$ era casada y $15,4 \%$ convivía); 40,3\% de ellas había cursado hasta Enseñanza Básica y 41,3\% hasta Enseñanza Media. Sesenta y seis como dos porciento no tenía un trabajo remunerado y $33,8 \%$ de ellas sí lo tenía (de estas últimas, 52,9\% era estable y $22,1 \%$ era ocasional). Las mujeres tenían un número de hijos que fluctuaba entre 0 y 10 , con una mediana en 2 . Respecto del número de hijos que vivían con ellas, el rango fluctuaba entre 0 y 6 , con una mediana de 2. La mayoría de las mujeres no participaba en ningún grupo social $(65,2 \%), 21,9 \%$ lo hacía frecuentemente en uno o más y $12,0 \%$ lo hacía en forma ocasional (Tabla 1).

\section{Diagnóstico de ingreso al tratamiento}

Para el diagnóstico de la intensidad del cuadro depresivo se tuvieron tres fuentes: el diagnóstico registrado en los documentos del programa, el diagnóstico hecho por las entrevistadoras basado en los criterios CIE-10 y los resultados en el IDB (Tabla 2).

Según los registros del programa, 78,9\% de las mujeres de la cohorte presentan una depresión moderada, $15,7 \%$ una depresión leve y $5,4 \%$ una depresión severa. Según los criterios CIE-10 usados por las entrevistadoras, $62,7 \%$ presentan una depresión moderada, $25,4 \%$ una depresión severa y $11,9 \%$ una depresión leve.

Al comparar el puntaje en el IDB con el diagnóstico hecho por los profesionales de los centros nos encontramos con que no hay concordancia $(\mathrm{p}=0,3955)$ y al comparar este mismo puntaje con el diagnóstico realizado por las entrevistadoras encontramos que la concordancia fue estadísticamente significativa $(\mathrm{p}<0,0001)$.
En la Tabla 3 se muestra la concordancia entre los diagnósticos hechos por los profesionales de los centros y el realizado por la entrevistadora. El índice de concordancia se obtiene con los casos que están en la diagonal (91/166), que corresponde a $54,8 \%$. La subestimación de la gravedad son los casos que quedan sobre esta diagonal (55/166) y corresponde a $33,1 \%$. La sobrestimación de la gravedad son los casos que caen bajo la diagonal (20/166) y corresponde a $12,1 \%$. Por tanto, se constató que hay problemas en el diagnóstico de la gravedad del cuadro clínico, con una tendencia a subestimarla.

\section{Tabla 1. Características socio-demográficas de la muestra de mujeres ingresadas al programa de tratamiento para depresión, en 7 Centros de APS ( $n=201)$}

\begin{tabular}{|c|c|}
\hline \multicolumn{2}{|l|}{ Variable } \\
\hline $\begin{array}{l}\text { Edad (años) } \\
\text { - } \quad \text { Rango } \\
\text { - } \quad \text { Promedio y desviación estándar }\end{array}$ & $\begin{array}{l}25 \text { a } 65 \\
44,0 \pm 11,0\end{array}$ \\
\hline $\begin{array}{l}\text { Escolaridad alcanzada } \\
\text { - } \quad \text { Sin estudios } \\
\text { - } \quad \text { E. Básica } \\
\text { - } \quad \text { E. Media } \\
\text { - } \quad \text { E. Técnica } \\
\text { - } \quad \text { E. Universitaria }\end{array}$ & $\begin{array}{c}2(1,0 \%) \\
81(40,3 \%) \\
83(41,3 \%) \\
25(12,4 \%) \\
10(5,0 \%)\end{array}$ \\
\hline $\begin{array}{l}\text { Estado civil } \\
\text { - Soltera } \\
\text { - } \text { Casada } \\
\text { - Conviviente } \\
\text { - Separada } \\
\text { - Viuda }\end{array}$ & $\begin{array}{l}31(15,4 \%) \\
96(47,8 \%) \\
31(15,4 \%) \\
33(16,4 \%) \\
10(5,0 \%)\end{array}$ \\
\hline $\begin{array}{l}\text { No de hijos que viven con ella } \\
\text { - Rango } \\
\text { - Mediana }\end{array}$ & $\begin{array}{l}0 \text { a } 6 \\
2\end{array}$ \\
\hline $\begin{array}{l}\text { Situación ocupacional } \\
\text { - } \quad \text { Sin trabajo remunerado } \\
\text { - } \quad \text { Con trabajo remunerado }\end{array}$ & $\begin{array}{r}133(66,2) \\
68(33,8)\end{array}$ \\
\hline $\begin{array}{l}\text { Participación en grupos sociales } \\
\text { - } \quad \text { No participa en grupos sociales } \\
\text { - Participa en forma ocasional } \\
\text { - } \quad \text { Participa en forma frecuente }\end{array}$ & $\begin{array}{r}131(65,1 \%) \\
26(12,0 \%) \\
44(21,9 \%)\end{array}$ \\
\hline
\end{tabular}


Evaluación del programa para depresión en atención primaria - R. Alvarado et al

Tabla 2. Características del diagnóstico en la muestra de mujeres ingresadas al programa de tratamiento para depresión, en 7 Centros de APS $(n=201)$

\begin{tabular}{|c|c|c|c|}
\hline Tipo de depresión & n (\%) & puntaje IDB & p-value \\
\hline \multicolumn{4}{|c|}{ Diagnóstico en el programa $(*)$} \\
\hline - Leve & $26(15,7 \%)$ & $24,3 \pm 8,5$ & \multirow{4}{*}{0,3955} \\
\hline - Moderado & $131(78,9 \%)$ & $24,4 \pm 9,4$ & \\
\hline - Severo & $9(5,4 \%)$ & $28,8 \pm 11,7$ & \\
\hline - Total & $166(100,0 \%)$ & & \\
\hline \multicolumn{4}{|c|}{ Diagnóstico de la entrevistadora } \\
\hline - Leve & $24(11,9 \%)$ & $14,8 \pm 6,8$ & \multirow{4}{*}{$<0,0001$} \\
\hline - Moderado & $126(62,7 \%)$ & $23,9 \pm 8,2$ & \\
\hline - Severo & $51(25,4 \%)$ & $32,3 \pm 7,6$ & \\
\hline - Total & $201(100,0 \%)$ & & \\
\hline
\end{tabular}

(*) Nota: Sólo se pudo encontrar los registros de ingreso en 166 de las 201 mujeres.

Tabla 3. Concordancia del diagnóstico entre el equipo profesional del Centro y la entrevistadora, en 166 mujeres ingresadas al programa de tratamiento para depresión, en 7 Centros de APS

\begin{tabular}{|lccc|}
\hline $\begin{array}{l}\text { Diagnóstico } \\
\text { según registros } \\
\text { de ingreso }\end{array}$ & \multicolumn{3}{c|}{$\begin{array}{l}\text { Diagnóstico según } \\
\text { la entrevistadora }\end{array}$} \\
\hline Leve & 2 & 19 & 5 \\
Leve & Moderada & Severa \\
Moderada & 16 & 84 & 31 \\
\hline Severa & 1 & 3 & 5 \\
\hline
\end{tabular}

\section{Tratamientos}

El tiempo transcurrido entre el diagnóstico y el inicio del tratamiento fluctuó entre 0 y 71 días, con un promedio en 9,5 días $(\mathrm{de}=14,0)$. Cuarenta y cinco como cuatro porciento de las mujeres inició su tratamiento de inmediato después de realizado el diagnóstico, 19,4\% lo hizo dentro de la primera semana y $35,2 \%$ parten con su tratamiento después de 1 semana de hecho el diagnóstico.

En $86,8 \%$ de las mujeres se indicó tratamiento farmacológico desde un inicio y en $13,2 \%$ no se hizo. Al explorar los diagnósticos en los registros del programa para los casos a los cuales no se indicó antidepresivos, se encontró que en 35,3\% eran definidas como depresión leve y en $64,7 \%$ como depresión moderada. Según el diagnóstico hecho por la entrevistadora, entre las que no recibieron fármacos había 16,0\% con una depresión leve y en- tre las que sí tuvieron esta indicación hubo 12,2\% de cuadros leves $(p=0,4611)$. No se observaron diferencias estadísticamente significativas en las puntuaciones en el IDB entre el grupo al que no se le indicó antidepresivos $(21,7 \pm 7,9$ puntos $)$ respecto del grupo al que sí se le indicó $(25,4 \pm$ $9,6$ puntos $)(\mathrm{p}=0,0748)$.

Respecto de la indicación de psicoterapia individual, en 53,5\% de estas mujeres no se indicó, en $12,8 \%$ sí se hizo pero ellas no acudieron, y en $33,7 \%$ se indicó y ellas asistieron al menos a una sesión. No se encontraron diferencias significativas respecto de la indicación de asistir a psicoterapia individual de acuerdo a la gravedad del cuadro depresivo, ni desde la perspectiva del diagnóstico hecha por el equipo del centro $(\mathrm{p}=0,4989)$, ni desde la perspectiva del diagnóstico hecho por la entrevistadora $(\mathrm{p}=0,2781)$, ni respecto de la puntuación en el IDB: $24,7 \pm 9,0$ puntos en el grupo donde no se indica y $25,2 \pm 10,3$ puntos en el grupo donde sí se indica $(\mathrm{p}=0,7738)$.

En cuanto a la indicación inicial de asistir a la intervención psicosocial de grupo, en $66,1 \%$ de las mujeres no se indicó participar en esta, en $12,5 \%$ sí se hizo pero ellas no fueron y en $21,4 \%$ se indicó y ellas asistieron al menos a una sesión. Al igual que en el caso de la psicoterapia individual, no se encontraron diferencias estadísticamente significativas respecto de la indicación de asistir a esta intervención grupal y la gravedad del cuadro depresivo, ni desde la perspectiva del diagnóstico registrado en el programa $(\mathrm{p}=0,1120)$, ni desde 
Evaluación del programa para depresión en atención primaria - R. Alvarado et al

Tabla 4. Variación en el puntaje del IDB en una muestra de 159 mujeres ingresadas al programa de tratamiento para depresión, según adherencia a sus controles a los 6 meses, en 7 Centros de APS

\begin{tabular}{|lcccc|}
\hline Grupo de seguimiento & $\mathbf{n}$ & ingreso & $\mathbf{3}^{\text {er }}$ mes & $\mathbf{6}^{\text {to }}$ mes \\
\hline Controles regulares & 70 & $23,6 \pm 8,7$ & $16,9 \pm 10,0$ & $14,3 \pm 9,7$ \\
\hline Controles irregulares & 31 & $30,9 \pm 8,9$ & $24,4 \pm 11,0$ & $19,2 \pm 13,9$ \\
\hline Abandono tratamiento & 58 & $21,8 \pm 7,7$ & $19,1 \pm 10,0$ & $18,9 \pm 10,9$ \\
\hline
\end{tabular}

la perspectiva del diagnóstico hecho por la entrevistadora $(\mathrm{p}=0,1368)$, ni en la puntuación en el IDB: $25,2 \pm 9,1$ puntos en el grupo donde no se indica y $26,3 \pm 10,3$ puntos en el grupo donde sí se indica $(\mathrm{p}=0,4570)$.

En síntesis, no se encontró asociación entre la intensidad del cuadro depresivo y la indicación de farmacoterapia, ni de psicoterapia individual, ni de participación en un grupo de apoyo psicosocial.

\section{Adherencia al programa de tratamiento}

A los 6 meses, $60,8 \%$ de todo el grupo se mantenía en control (101 casos) y 34,9\% había abandonado en algún momento de este período (58 casos). Dentro del primer grupo, 70 mujeres $(42,2 \%)$ seguían con sus controles en forma regular (había acudido a más de $90 \%$ de sus citas) y $31(18,6 \%)$ lo hacían en forma irregular (había acudido a menos del $90 \%$ de sus citas). En este período de tiempo, sólo un caso fue derivado a un centro de mayor complejidad $(0,6 \%)$ y seis de ellas se habían dado de alta del programa $(3,6 \%)$.

\section{Evolución de la intensidad del cuadro depresivo}

En la Tabla 4 se muestra el puntaje promedio y su desviación estándar en el IDB, para cada uno de los grupos en los 3 momentos de la evaluación. Dentro del grupo con controles regulares el porcentaje de descenso en el IDB fue de 35,0\% (desde un promedio en 23,6 puntos a un promedio de 14,3 puntos, a los 6 meses) y en el grupo de controles irregulares fue de 37,8\% (desde 30,9 puntos a 19,2 puntos). En cambio, en el grupo que abandonó el programa el descenso sólo fue de $13,3 \%$ (desde 21,8 puntos a 18,9 puntos). Estas diferencias fueron estadísticamente significativas en el análisis de varianza $(\mathrm{p}=0,0002)$. El análisis de comparaciones múltiples (técnica de Bonferroni) mostró que había diferencias significativas entre el grupo de controles regulares con el que abandona $(\mathrm{p}=0,0003)$ y de este último con el de controles irregulares $(p=0,0006)$. En cambio, no hubo diferencias significativas entre los dos grupos que se mantuvieron en control ( $\mathrm{p}=0,4357)$.

Por tanto, los dos grupos que se mantienen en control -independientemente de que este sea en forma regular o irregular-, logran una reducción significativamente mayor en la puntuación del IDB respecto del grupo que abandona, quienes muestran una tasa de descenso que es sólo $1 / 3$ de la observada en los otros dos grupos.

El grupo con controles regulares logró gran parte del descenso en los primeros tres meses: $28,4 \%$ (desde 23,6 puntos a 16,9 puntos); en contraste con el grupo que mantuvo sus controles en forma irregular donde el descenso fue de 22,3\% (desde 30,9 puntos a 24,4 puntos). Esta diferencia no fue estadísticamente significativa.

\section{Discusión}

Este trabajo corresponde a la segunda evaluación que se realizó del "Programa para la Detección, Diagnóstico y Tratamiento Integral de la Depresión en Atención Primaria" en Chile; la primera se efectuó durante la fase de puesta en marcha entre los años 2001 y 2002, en centros que estaban interesados en este problema y con equipos motivados, observando que los tratamientos eran efectivos en reducir los síntomas depresivos ${ }^{14}$. En esta segunda ocasión se realizó en un contexto donde el programa se había extendido a todo Chile, los equipos ya llevaban al menos un año de funcionamiento, se utilizaron entrevistas clínicas estandarizadas para la clasificación de los casos (basada en los criterios CIE-10) y se realizó un seguimiento de 6 meses.

La principal debilidad metodológica de este estudio es la falta de aleatorización en la asignación de los casos al programa de tratamiento v/s una atención de control. Pero esto resulta técnicamente 
imposible dado que el programa de tratamiento se encuentra funcionando en todo el país y reñido con la ética ya que no se puede otorgar servicios de menor calidad a un sub-grupo de estos pacientes. Es por esta razón que en la actualidad se aceptan cada vez más los estudios para evaluación de intervenciones - o conjuntos de estas -, utilizando diseños naturalísticos y cuasi-experimentales (como en este caso), y que tienen la ventaja adicional de lograr una mayor validez externa, es decir, que sus resultados se aproximan más a lo que sucede en las condiciones reales en que se entregan los servicios $^{18,19}$.

Otra debilidad de este estudio fue la pérdida de $17,4 \%$ de los casos durante el seguimiento. Se realizó un análisis para verificar si existían diferencias de este grupo respecto del que se mantuvo en el seguimiento, no encontrándose diferencias estadísticamente significativas para el estado civil, la escolaridad, la existencia de trabajo remunerado, la participación en grupos sociales, ni para el diagnóstico al ingreso (hecho por la entrevistadora), ni para el puntaje en el IDB. Sólo se encontraron diferencias en la edad: el grupo que continúa en el tratamiento tenía un promedio de edad algo mayor al grupo que abandonaba $(45,0 \pm 10,5$ vs $39,2 \pm$ 12,2 , con $p=0,0040$ ). Esta diferencia entre ambos grupos no parece introducir un sesgo relevante, ya que según el conocimiento actual estas diferencias de edad no se relacionarían fuertemente con la gravedad del cuadro clínico, ni con los resultados de los tratamientos.

Respecto de los objetivos del estudio, nuestros resultados confirman que el programa de tratamiento que se brinda en estos centros de atención primaria es efectivo, lo que coincide con los resultados del estudio previo ${ }^{14} \mathrm{y}$ con lo reportado en otros países de mayores ingresos ${ }^{7-10}$. También es importante señalar que reafirma los resultados del ensayo clínico comunitario realizado en nuestro medio ${ }^{11}$, lo que implica una ampliación de la validez externa de sus resultados.

Cabe destacar que los mejores resultados se obtuvieron en el sub-grupo que realiza sus controles en forma regular (acude a más de $90 \%$ de sus citas). Aunque la diferencia respecto del grupo con controles irregulares (acude a menos de 90\% de sus citas) no fue estadísticamente significativa, estos resultados sugieren que existe una "relación dosis-respuesta”, lo que refuerza aún más la existencia de una buena efectividad de este programa.
A modo de síntesis inicial, cabe señalar que el demostrar la efectividad de un programa de este tipo no sólo es importante para Chile y el desarrollo de sus políticas en salud mental, sino también porque reafirma que esta estrategia basada en programas que se realizan en atención primaria es válida y útil para otros países en vías de desarrollo ${ }^{20-25}$.

Por otra parte, se encontraron tres aspectos que son debilidades de este programa y que requerirá tomar medidas por parte de las autoridades correspondientes: 1) no se está realizando en forma correcta el diagnóstico de la gravedad del cuadro clínico, con una tendencia a la sub-calificación de esta gravedad; 2) no se están aplicando los diferentes tratamientos (farmacoterapia, psicoterapia y grupo de apoyo psicosocial) en forma escalonada de acuerdo a la gravedad del cuadro clínico y en base a las recomendaciones de la guía de práctica clínica; y 3) existe un porcentaje elevado de abandono de los tratamientos. Los dos primeros aspectos deberían ser mejorados a través de un entrenamiento permanente de los equipos clínicos que manejan este programa en los centros de atención primaria y dada la alta rotación de algunos de sus profesionales, será necesario mantener actividades de capacitación que sean continuas en el tiempo ${ }^{9,26,27}$. El tercer aspecto, que se refiere al abandono de los tratamientos, es un problema frecuente en todas las enfermedades que tienen un curso prolongado o crónico (tales como diabetes o HTA) y es uno de los principales factores que afecta la efectividad poblacional de estos programas de tratamiento. Para mejorarlo es necesario incorporar técnicas que han demostrado mejorar la adherencia, tales como seguimiento telefónico y educación específica para esto, así como el uso de medidas de rescate de pacientes (visita en el domicilio, contactos con familiares, etc.). Hay experiencias con buenos resultados, tanto en el extranjero ${ }^{28-30}$, como en nuestro país ${ }^{31}$.

A raíz de nuestros resultados, en estudios futuros debería mejorarse la comparabilidad entre los grupos, ya sea a través de optimizar la asignación de los casos en el diseño o incorporar técnicas de análisis estadístico para controlar el potencial sesgo. También debería estudiarse la situación específica de los hombres que inician su tratamiento por depresión, ya que en ellos los resultados podrían ser diferentes ${ }^{13}$. Nuestro estudio mostró que se obtenían buenos resultados a pesar de que 
Evaluación del programa para depresión en atención primaria - R. Alvarado et al

la indicación de intervenciones no se ajustaba a la gravedad del cuadro clínico, lo que hace necesario estudiar la efectividad específica de cada una de estas intervenciones y su particular aporte al resultado global en estos pacientes. Finalmente, para reducir el abandono de los tratamientos sería de interés estudiar la posible existencia de sub-grupos con un perfil de riesgo mayor, lo que permitiría concentrar acciones en ellos (tales como el seguimiento telefónico u otra medida).

\section{Referencias}

1. Vicente B, Rioseco P, Saldivia S, Kohn R, Torres S. Estudio chileno de prevalencia de patología psiquiátrica (DSM-III-R / CIDI). Rer Med Chile 2002; 130: 527-36.

2. Araya R, Rojas G, Fritsh R, Acuña J, Lewis G. Common Mental Disorders in Santiago, Chile: Prevalence and socio-demographic correlates. Br J Psychiatry 2001; 178: 228-33.

3. Concha M. La carga de enfermedad en Chile. Chile: Ministerio de Salud; 1996.

4. Goldberg DP, Lecubrier Y, Form and frequency of mental disorders across centres. En: Üstün TB, Sartorius N, eds., Mental illness in general health care: an international study. Chichester, John Wiley \& Sons, 1995.

5. Geddes J, Butler R, Hatcher S. Depressive disorders. En: BMJ Publishing Group. Clinical Evidence Mental Health. 2002. Pp. 84-106.

6. UK Department of Health. Treatment choise in psychological therapies and couselling. Evidence Based Clinical Practice Guideline. Depatment of Health Publications. London, United Kingdom. 2001.

7. Katon W, Von Korff M, Lin E, Walter E, Simon GE, Bush $\mathrm{T}$, et al. Collaborative management to achieve treatment guidelines. Impact on depression in Primary Care. JAMA 1995; 273: 1026-31.

8. Sherbourne CD, Wells KB, Duan N, Miranda J, Unützer J, Jaycox L, et al. Long - term effectiveness of Diseminating Quality Improvement for depression in Primary Care. Arch Gen Psychiatry 2001; 58: 696-703.

9. Gilbody S, Whitty P, Grimshaw J, Thomas R. Educational and organizacional interventions to improve the management of depression in Primary Care. JAMA 2003; 289: 3145-51.

10. Badamgarav E, Weingarten SR, Henning JM, Knight K, Hasselblad V, Gano A, et al. Effectiveness of Disease Management Programs in Depression: a Systematic Review. Am J Psychiatry 2003; 160: 2080-90.

11. Araya R, Rojas G, Fritsh R, Gaete J, Rojas M, Simon G, et al. Treating depression in primary care in low-income women in Santiago, Chile: a randomised controlled trial. Lancet 2003; 361: 995-1000.

12. Chile, Ministerio de Salud. Guía Clínica para la Atención Primaria. La depresión: detección, diagnóstico y tratamiento. Santiago; Unidad de Salud Mental y Departamento de Modelo de Atención, del Ministerio de Salud; 2001.

13. Addis ME. Gender and depression in men. Clin Psychol Sci Prac 2008; 15: 153-68.

14. Alvarado R, Vega J, Sanhueza G, Muñoz M. Evaluación del Programa para la Detección, Diagnóstico y Tratamiento Integral de la Depresión en atención primaria, en Chile. Rev Panam Salud Pública 2005; 18: 278-86.

15. Beck AT, Ward CH, Mendelson M, Mock JE, Erbaugh J. An inventory of measuring depression. Arch Gen Psychiatry 1961; 4: 561-85.

16. Beck AT, Steer RA. Beck Depression Inventory (BDI). En: American Psychiatric Association (ed). Handboock of Psychiatric Measures. Washington DC, USA. 2000. Pp. 519-23.

17. Beck AT, Steer RA, Gabin MG. Psychometric properties of the Beck Depression Inventory: twenty-five years of evaluation. Clinical Psychology Review 1988; 8: 78-100.

18. Hendricks Brown C, Ten Have TH, Jo B, Dagne G, Wyman P, Muthén B, et al. Adaptative designs for randomized trials in public health. Annu Rev Public Health 2009; 30: 1-25.

19. Campbell D, Stanley J. Diseños experimentales y cuasiexperimentales en la investigación social. Buenos Aires, Argentina. Ed. Amorrortu; 1973.

20. Berwick DM. Lessons from developing nations on improving health care. BMJ 2004; 328: 1124-9.

21. Evans T, Nishtar S, Atun R, Etienne C. Scaling up research and learning for health systems: time to act. The Lancet 2008; 372: 1529-31.

22. Gruen RL, Elliott JH, Nolan MJ, Lawton PD, Parkhill A, McLaren CJ, et al. Sustainability science: an integrated approach for health-programme planning. The Lancet 2008; 372: 1579-89.

23. Patel V, Araya R, Chatterjee R, Chisholm D, Cohen A, De Silva $\mathrm{M}$, et al. Treatment and prevention of mental disorders in low-income and middle-income countries. The Lancet 2007; 370: 991-1005.

24. Saraceno B, van Ommeren M, Batniji R, Cohen A, Gureje O, Mahoney J, et al. Barriers to improvement of mental health services in low-income and middleincome countries. The Lancet 2007; 370: 1164-74.

25. Lancet Global Mental Health Group. Scale up services for mental disorders: a call for action. The Lancet 2007; 370: 1241-52.

26. Rutz W, Walinder J, Eberhard G, Holmberg G, von Kno- 
Evaluación del programa para depresión en atención primaria - R. Alvarado et al

rring AL, von Knorring L, et al. An educational program on depressive disorders for general practitioners on Gotland: background and evaluation. Acta Psychiatr Scand 1989; 79: 19-26.

27. Thompson C, Kinmonth AL, Stevens L, Peveler RC, Stevens A, Ostler KJ, et al. Effects of a clinical-practice guideline and practice-based education on detection and outcome of depression in Primary Care: Hampshire Depression Project ramdomised controlled trial. Lancet 2000; 355: 185-91.

28. Katon W, Rutter C, Ludman EJ, Von Korff M, Lin E, Simon GE, et al. A ramdomised trial of relapse prevention of depression in Primary Care. Arch Gen Psychiatry 2001; 58: 241-7.
29. Weingarten SR, Henning JM, Badamgarav E, Knight K, Hasselblad V, Gano A, et al. Interventions used in disease management programmes for patients with chronic illness-which ones work? Metaanalysis of published reports. BMJ 2002; 325: 925-32.

30. Rost K, Nutting P, Smith JL, Elliott CE, Dickinson M. Managing depression as a chronic disease: a randomized trial of ongoing treatment in primary care. BMJ 2002; 325: 934-9.

31. Fritsch R, Araya R, Solís J, Montt ME, Pilowsky D, Rojas G. [A randomized trial of pharmacotherapy with telephone monitoring to improve treatment of depression in primary care in Santiago, Chile]. Rev Med Chile 2007; 135: 587-95. 